\title{
Getting to know the neighbourhood
}

\section{Cff \\ a parenchymal
component \\ that responds \\ to and is \\ shaped by \\ disseminated \\ cancer cells}

Our understanding of what constitutes the metastatic microenvironment or niche, particularly at early stages of metastatic colonization, has been hindered by technical obstacles with spatially isolating distinct cell populations from the bulk tissue. Ombrato et al. reasoned that there might be previously uncharacterized cell types present in the metastatic niche at low frequency, which undergo changes upon the arrival of disseminated cancer cells. To investigate this, they developed a fluorescent labelling system, which uses the metastatic cells themselves to directly mark the neighbouring niche cells in vivo.

To explore the early changes driven by metastatic breast cancer cells within the surrounding tissue of the lung, the authors engineered $4 \mathrm{~T} 1$ breast cancer cells to co-express GFP and a secreted fluorescent mCherry protein fused to a lipid-soluble tag (sLP-mCherry) to enable cell penetration. In vitro, these labelling-4T1 cells secrete sLP-mCherry, which in turn is taken up by other labelling-4T1 cells as well as by nearby unlabelled cells where it resides in lysosomal-like structures for approximately 48 hours. In an experimental metastasis model wherein labelling-4T1 cells $\left(\mathrm{GFP}^{+} \mathrm{mCherry}{ }^{+}\right)$ are injected intravenously into the tail vein of syngeneic mice to form lung metastases, this labelling system was shown to effectively mark proximate host tissue cells (GFP-mCherry ${ }^{+}$). Subsequent isolation of the cell populations by fluorescence-activated cell sorting (FACS) enabled a spatial map of the metastatic niche to be reconstituted and functional testing of the labelled cells to be carried out together with unlabelled distal cells from the same lung (GFP- ${ }^{-}$Cherry $\left.{ }^{-}\right)$.

The authors began by using their labelling system to look for components previously implicated in local metastatic niche formation, thereby validating the approach. This led to the identification of CD $45^{+}$immune cells, which were predominantly of myeloid lineage, and included lung neutrophils. Further examination of $\mathrm{mCherry}^{+}$-niche neutrophils uncovered that this subpopulation had enhanced translation, oxidative phosphorylation and levels of reactive oxygen species (ROS) consistent with a protumorigenic phenotype relative to unlabelled neutrophils from the same lung.

Next, the authors focused their attention on other non-immune components of the metastatic microenvironment. To observe how these cell types changed during the progression from establishment of micrometastases to advanced macrometastases, gene expression profiles were generated at different time points. A large number of changes occurred early and included alterations in pathways ranging from proliferation, inflammation and tissue remodelling. Unexpectedly, changes in pathways associated with lung epithelial cells were also observed, suggesting the lung parenchyma could contribute to formation of the metastatic niche. In line with this, $\mathrm{mCherry}^{+}$-niche cells expressing the epithelial cell adhesion marker EPCAM were detected in the metastatic microenvironment and were found to have an increased proliferative capacity compared with their unlabelled counterparts.

Single cell RNA sequencing of mCherry ${ }^{+}$-niche cells further defined the non-immune compartment of the metastatic niche revealing distinct clusters of stromal cells as well as two clusters of Epcam ${ }^{+}$epithelial cells differentiated by the expression of $C d h 1$, the gene encoding E-cadherin. Notably, the Epcam ${ }^{+} \mathrm{Cdh1}^{-}$epithelial cells, which formed the majority of the epithelial cell population in the metastatic niche, displayed a de-differentiated, progenitor phenotype.

Using ex vivo assays, the authors were able to show that mCherry ${ }^{+}$ $\mathrm{EPCAM}^{+}$epithelial cells when cocultured with $\mathrm{CD} 31^{+}$endothelial cells typically formed bronchiolar organoids in contrast to unlabelled lung epithelial cells, which generated alveolar organoids, demonstrating the multi-lineage differentiation potential of these cancer-associated parenchymal cells. In addition, these niche cells possess high self-renewal ability and likely originate from alveolar type II (AT2) cells.

As alveolar cell clusters with increased proliferative capacity were observed in human breast cancer lung metastases, it will be interesting to see if this strategy, when applied to other metastatic microenvironments in mice, similarly reveals a parenchymal component that responds to and is shaped by disseminated cancer cells.

Anna Dart

ORIGINAL ARTICLE Ombrato, L. et al. Metastatic-niche labelling reveals parenchymal cells with stem features. Nature 572, 603-608 (2019) 